\title{
Bulk Micromegas detectors for large TPC applications
}

\author{
J. Bouchez ${ }^{\text {a }}$, D. R. Burke ${ }^{\text {a }}$, Ch. Cavata ${ }^{\text {a, }}$, . Colas ${ }^{\text {a }}$, \\ X. De La Broise ${ }^{\text {a }}$, A. Delbart ${ }^{\text {a }}$, A. Giganon ${ }^{\text {a }}$, I. Giomataris ${ }^{\text {a }}$, \\ P. Graffin ${ }^{\text {a }}$, J.-Ph. Mols ${ }^{\text {a }}$, F. Pierre ${ }^{\text {a }}$, J.-L. Ritou ${ }^{\text {a }}$, A. Sarrat ${ }^{\text {a }}$, \\ E. Virique ${ }^{\mathrm{a}}$, M. Zito ${ }^{\mathrm{a}, *}$, E. Radicioni $^{\mathrm{b}}$, R. De Oliveira ${ }^{\mathrm{c}}$, \\ J. Dumarchez ${ }^{\mathrm{d}}, \mathrm{N}$. Abgrall $^{\mathrm{e}}, \mathrm{P}$. Bene $^{\mathrm{e}}$, A. Blondel $^{\mathrm{e}}$, A. Cervera $^{\mathrm{e}, \mathrm{g}}$, \\ D. Ferrere ${ }^{\mathrm{e}}$, F. Maschiocchi ${ }^{\mathrm{e}}$, E. Perrin ${ }^{\mathrm{e}}$, J.-P. Richeux ${ }^{\mathrm{e}}$,

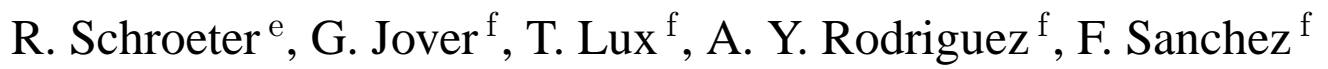 \\ ${ }^{a}$ Dapnia, CEA-Saclay, 91191 Gif-Yvette, France \\ ${ }^{\mathrm{b}}$ INFN Sezione di Bari, Bari, Italy \\ ${ }^{\mathrm{c}}$ CERN/TS-DEM, CH 1211 Geneva, Switzerland \\ ${ }^{\mathrm{d}}$ LPNHE, Université Paris-VI-VII, Paris, France \\ ${ }^{\mathrm{e}}$ DPNC, Section de Physique, University of Geneva CH 1211 Geneva 4, Switzerland \\ ${ }^{\mathrm{f}} U A B$, IFAE, Bellaterra E-08193, Barcelona, Spain \\ $\mathrm{g}$ now at University of Valencia, Spain
}

\begin{abstract}
A large volume TPC will be used in the near future in a variety of experiments including $\mathrm{T} 2 \mathrm{~K}$. The bulk Micromegas detector for this TPC is built using a novel production technique particularly suited for compact and robust low mass detectors. The capability to pave a large surface with a simple mounting solution and small dead space between modules is of particular interest for these applications. We have built several large bulk Micromegas detectors $\left(27 \times 26 \mathrm{~cm}^{2}\right)$ and we have tested them in the former HARP field cage setup with a magnetic field. Cosmic ray data have been acquired in a variety of experimental conditions. Good detector performances and space point resolution have been achieved.
\end{abstract}

Key words: Micromegas, TPC

* corresponding author: zito@ hep.saclay.cea.fr 


\section{Introduction}

A large volume TPC will be used in the near future for a variety of experiments, including T2K, and it is envisaged for a Linear Collider detector. In the case of the T2K near detector, three large TPC will be built to measure the tracks produced by the interactions of a neutrino beam with peak energy around $600 \mathrm{MeV}$. The area to be instrumented corresponds to almost $10 \mathrm{~m}^{2}$ and a pad size around $70 \mathrm{~mm}^{2}$ is considered. Of course the pad size depends on the specific application. In our case it has been chosen taking into account several factors, from physics requirements to readout complexity and cost.

For the TPC readout plane, the "bulk" Micromegas technology [1] is the best technical solution to minimize the dead zones on the edges of the modules and to improve the gas gain uniformity. Moreover, this technique is suited to industrialization and mass production, thereby offering the perspective of a cheap alternative to wire chambers.

This technique for manufacturing Micromegas detectors in a single process has been developed in 2004 by a CERN-Saclay collaboration and is described in detail in Ref. [1]. A woven mesh is laminated on a Printed Circuit Board (PCB) covered by a photo-imageable film. Such a detector 'all-in one', called 'bulk' Micromegas, is robust and allows large areas, up to $50 \times 50 \mathrm{~cm}^{2}$ or more, to be made in one piece. At the end of the process, the micromesh is sandwiched between 2 layers of insulating material. The detector undergoes then UV exposure with an appropriate mask and chemical development. A few millimeter wide border at the edge can thus be produced and avoids the need of an external additional frame to support the stretched micromesh.

In the following section we describe the detectors that we have designed and built. Section 3 documents the experimental setup for the TPC tests at CERN. The results of these tests are reported in section 4 .

\section{The bulk Micromegas detector}

One end plate for the T2K TPC has dimensions of approximately $0.7 \times 2 \mathrm{~m}^{2}$. In our design, this large surface, which is typical of other applications, is segmented with detector units of area around $30 \times 30 \mathrm{~cm}^{2}$ that can be easily replaced. In particular, no service or connection is needed from the inner volume of the TPC.

Following these design considerations, four bulk Micromegas detectors have been built in the CERN/TS-DEM PCB production facility. The PCB, segmented into 1020 pads, $8 \times 8 \mathrm{~mm}^{2}$, with a $100 \mu \mathrm{m}$ insulation between them (fig. 1) is $3 \mathrm{~mm}$ 
thick. It comprises two layers of FR4 with blind vias in the inner layer (fig. 2). This solution avoids the gas tightness problems arising from the conventional two layers structure with vias sealed with epoxide resins.

The pads are arranged in 32 rows of 32 pads for an active surface of $26.76 \times 26.35 \mathrm{~cm}^{2}$. In one corner a 4 pads equivalent surface is reserved for the Micromesh high voltage supply connection from the backside of the PCB. A half-pad staggering from one row to the next assures a better charge-sharing between adjacent pads and therefore a more uniform track reconstruction performance for high momentum vertical tracks. The top conductive layer is realized with $12 \mu \mathrm{m}$ thick copper deposited on FR4.

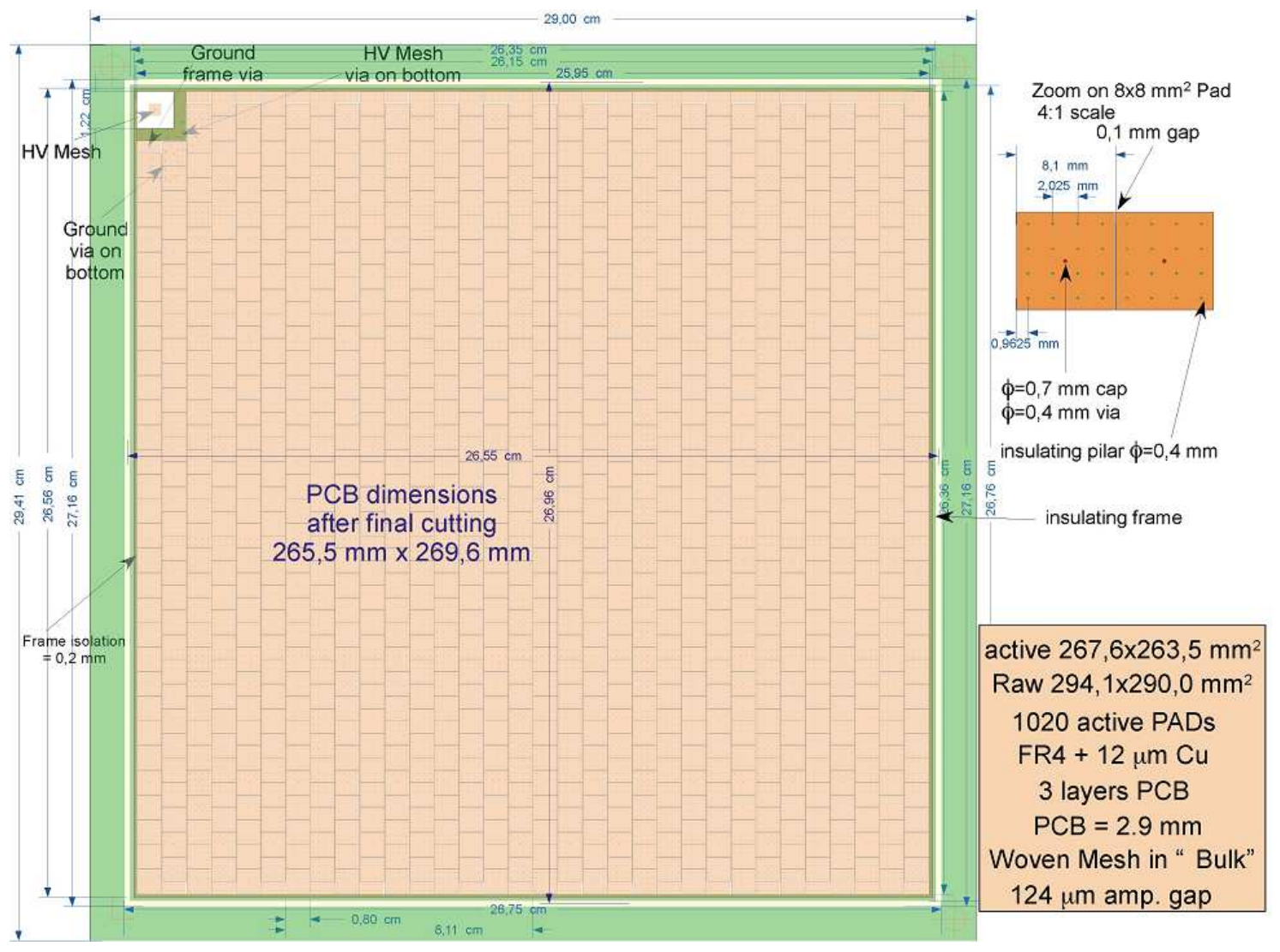

Fig. 1. View of the PCB from the anode pads side.

A sandwich of two layers of $64 \mu \mathrm{m}$ Pyralux PC1025 Photoimageable coverlay by DuPont [3], a woven micromesh and finally a layer of Pyralux is laminated on the PCB. The woven micromesh [2], is built out of $19 \mu \mathrm{m}$ thick 304L stainless steel wires. After weaving its thickness is reduced by $20-30 \%$ by a lamination process. The wires are spaced with a pitch of $59 \mu \mathrm{m}$. After photo-imaging, the mesh is held in place by the coverlay frame and 16320 regularly distributed $0.4 \mathrm{~mm}$ diameter cylindrical pillars, maintaining the amplification gap of $128 \mu \mathrm{m}$.

After protecting the sensitive detector surface with a melamine plate, the outer coverlay and PCB frame is cut to $3 \mathrm{~mm}$ in order to reduce the inactive area. Finally, 


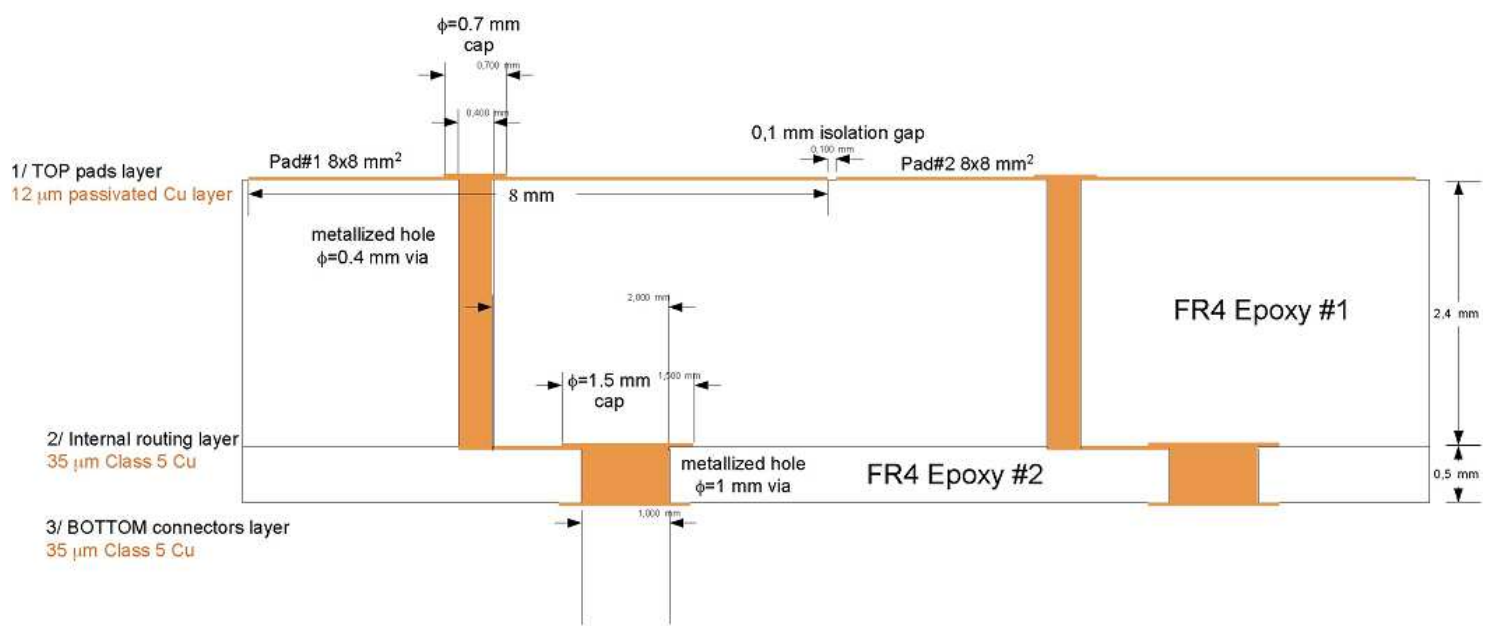

Fig. 2. The PCB cross-section.

the detector is glued onto an Aluminum support structure (fig. 3) to reinforce the PCB mechanical rigidity and to assure a $0.1 \mathrm{~mm}$ planarity tolerance. This structure hosts the seal for gas tightness.

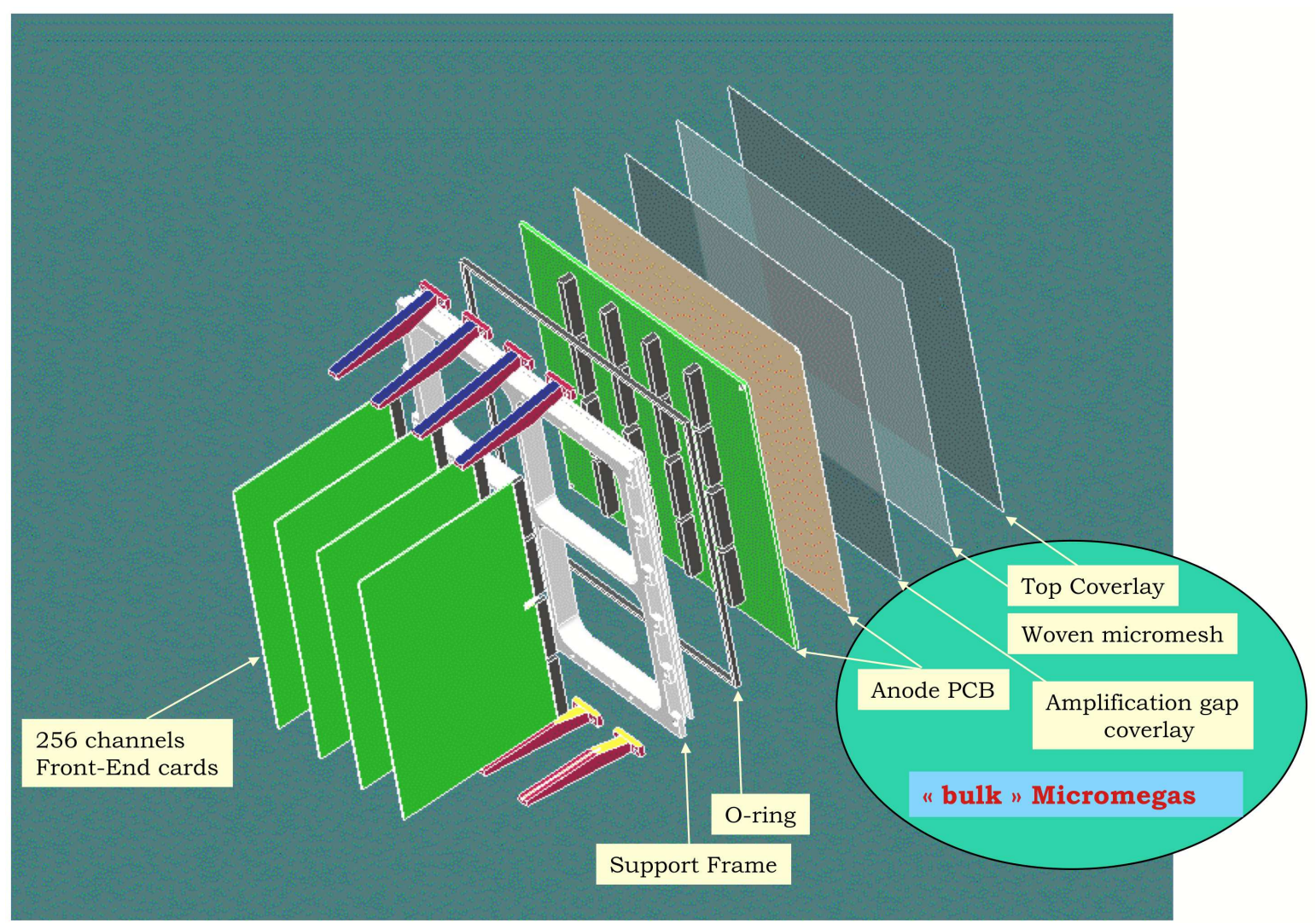

Fig. 3. Exploded 3D view of a Micromegas Module.

After the production, the detectors have been tested, first in air and then in a gas box. A good quality detector must stand in air a high voltage of $700 \mathrm{~V}$ drawing a current of a few nA. During this process, some defective pads (a few per mille) had 
to be disconnected.

The gas box provided an easy way to test the detector in a specific gas mixture with a ${ }^{55} \mathrm{Fe}$ source. The box was provided with a mylar window and an electrode to create a drift electric field pushing electrons toward the Micromegas mesh. The energy resolution was measured to be $10 \%$ for a $5.9 \mathrm{KeV}$ X-ray from the width of the Fe line (fig. 4). Several gas mixtures were tested with maximum gains ranging from a few thousand $\left(\mathrm{Ar}-\mathrm{CO}_{2}, \mathrm{Ar}-\mathrm{CF}_{4}\right)$ to a few $10^{4}$ in $\mathrm{Ar}-\mathrm{CF}_{4}-\mathrm{iC}_{4} \mathrm{H}_{10}$. For the latter mixture, the small Isobutane fraction, below the flammability limit, provides the required stability of operation while keeping all the interesting properties of an Argon- $\mathrm{CF}_{4}$ mixture: large drift velocity, low diffusion coefficient even in low magnetic field and long lifetime of the drifting electrons.
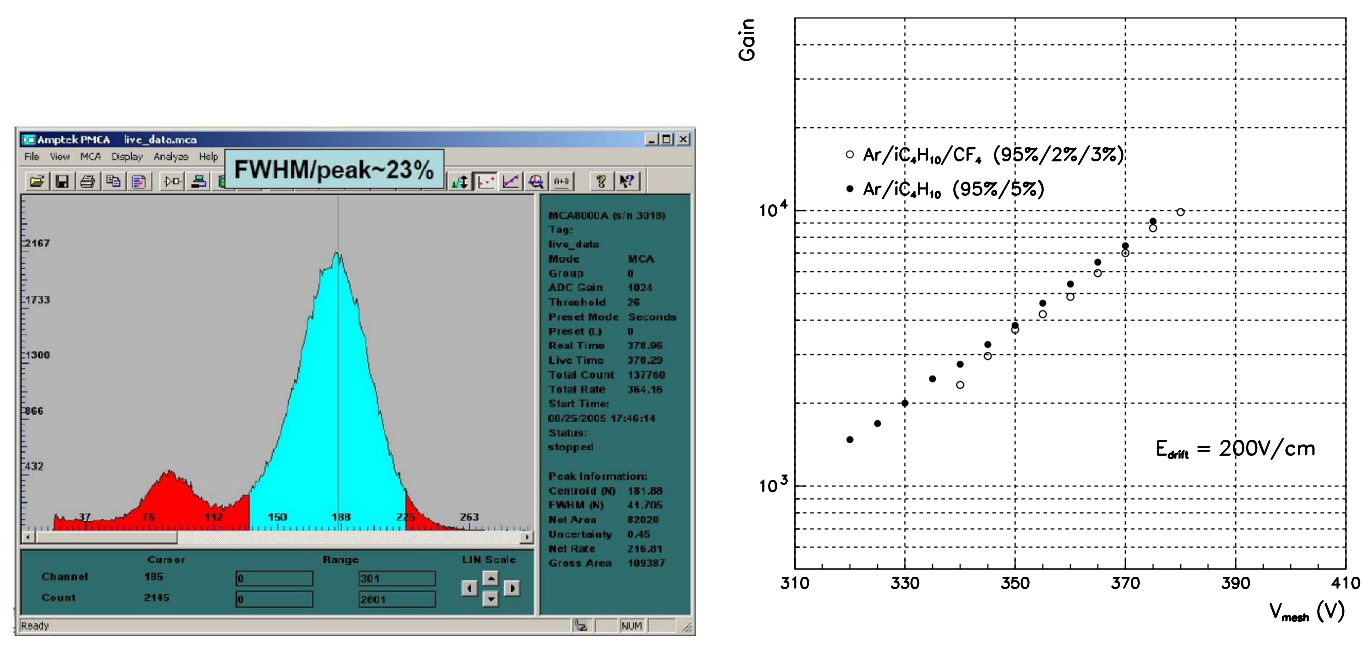

Fig. 4. Left: charge spectrum obtained with a Fe55 X ray source showing a resolution of $10 \%$. Right: gain versus micromesh high voltage in $\mathrm{Ar}^{-} \mathrm{iC}_{4} \mathrm{H}_{10}(5 \%)$ (black dots) and $\mathrm{Ar}-\mathrm{CF}_{4}(3 \%)-\mathrm{iC}_{4} \mathrm{H}_{10}(2 \%)$ (open circles).

\section{The TPC experimental setup}

The TPC experimental setup at CERN consists of the former HARP TPC field cage inside a solenoidal magnet and its gas system, read out by the Alice TPC $10 \mathrm{MHz}$ electronics. The TPC field cage is described in more details in [4]. It consists of a large cylindrical vessel, $80 \mathrm{~cm}$ inner diameter and $154.1 \mathrm{~cm}$ in drift length, where a series of strips connected by a resistor chain creates an axial electric field. The cathode was set at a potential corresponding to an electric field of $160 \mathrm{~V} / \mathrm{cm}$. The TPC field cage is mounted inside a solenoidal magnet of $90 \mathrm{~cm}$ inner diameter and $225 \mathrm{~cm}$ length.

The end-plate hosting two Micromegas modules (fig. 5) consists of an aluminum 
support structure covered on its inner surface by a large PCB with copper coating for an uniform termination of the drift electric field. The total inactive region between the two Micromegas sensitive regions is $12 \mathrm{~mm}$.

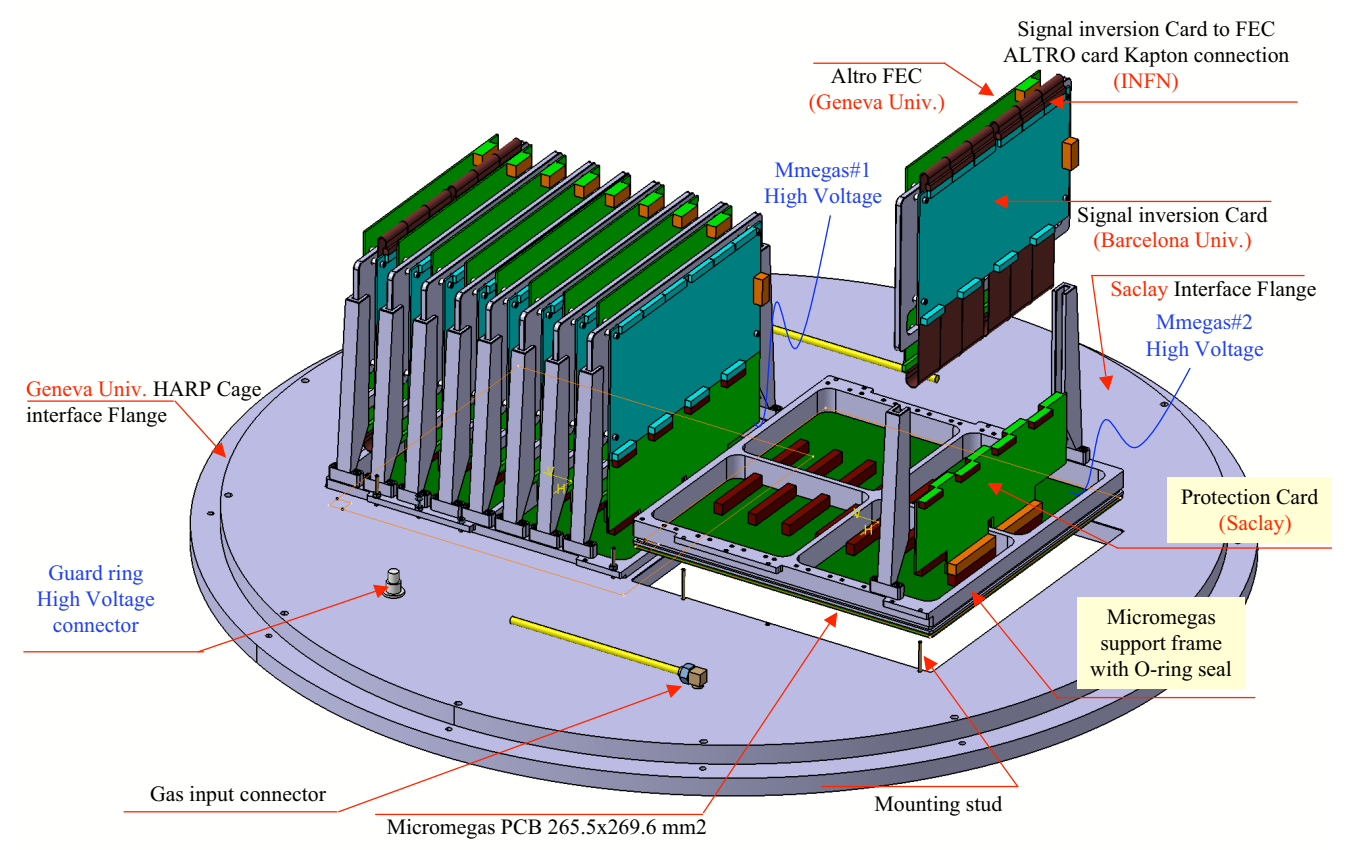

Fig. 5. The TPC endplate with two Micromegas detectors and the readout electronics.

The tests have been performed with the gas mixture $\mathrm{Ar}_{-} \mathrm{CF}_{4}(3 \%)-\mathrm{iC}_{4} \mathrm{H}_{10}(2 \%)$. It offers a fairly large drift velocity $(6.5 \mathrm{~cm} / \mu \mathrm{s})$ close to its maximum at this electric field, low diffusion coefficient at small magnetic field $(247 \mu \mathrm{m}$ for $\mathrm{B}=0.2 \mathrm{~T})$ and allows operation of Micromegas with a gain larger than 10000.

A system of seven scintillators, above and below the magnet, equipped with PMT, provided the trigger signal. The electronics consists of 12 invertor boards and 12 Alice TPC Front End Cards cards [5] for a total of 1536 channels. The cards instrumented the central part of the active region of the two detectors, for a total vertical length of $38.4 \mathrm{~cm}$ over 48 rows of pads. The input noise level was $2000 e$ RMS for a sampling frequency of $10 \mathrm{MHz}$, a shaping time of $190 \mathrm{~ns}$ and a charge to ADC conversion factor of $1000 e$ per ADC.

\section{Results}

We have taken cosmic ray data with this setup with the $\mathrm{Ar}-\mathrm{CF}_{4}(3 \%)-\mathrm{iC}_{4} \mathrm{H}_{10}(2 \%)$ gas mixture for almost one month. Typical Micromegas voltages were 330-360 V corresponding to gains between 2000 and 10000. The magnetic field varied from 0 
to $0.4 \mathrm{~T}$. The detector operated smoothly, the typical current drawn from the power supply being $1 \mathrm{nA}$. We observed sporadic sparks, triggering the current limit of the detector high voltage power supply (500 nA), without consequence neither for the detector nor for the electronics.

Data taken with cosmic rays have been analyzed using a complete analysis chain including an event display, a reconstruction program and a full simulation based on the Geant4 package [6]. A threshold equal to 3.5 times the RMS of the pedestal (typically $2 \mathrm{ADC}$ ) is applied to the raw data.

Hits on pads in the same row are associated in a cluster. In the case of clusters with two pads, the position cannot be estimated using a simple barycenter as the pad width is much larger than the electron cloud size, introducing large non-linearities. If the charge $q\left(q^{\prime}\right)$ has been collected on the pad extending from $x_{p}$ to $x_{p}+\Delta_{p}$ $\left(x_{p}-\Delta_{p}\right.$ to $\left.x_{p}\right)$, then

$$
\frac{q}{q+q^{\prime}}=\frac{\int_{x_{p}}^{x_{p}+\Delta_{p}} G\left(u, x_{t}, w_{t}\right) d u}{\int_{x_{p}}^{x_{p}+\Delta_{p}} G\left(u, x_{t}, w_{t}\right) d u+\int_{x_{p}-\Delta_{p}}^{x_{p}} G\left(u, x_{t}, w_{t}\right) d u},
$$

where $G\left(x, x_{t}, w_{t}\right)=\frac{1}{\sqrt{2 \pi} w_{t}} e^{-\frac{\left(x-x_{t}\right)^{2}}{2 w_{t}^{2}}}$ represents the probability density function of the primary electron cloud reaching the pad, neglecting the angle of the track with respect the normal to the measured coordinate $x$. The gaussian width $w_{t}$ is estimated from $w_{t}=C_{t} \sqrt{l_{d}}$, where $C_{t}$ is the transverse diffusion coefficient computed using the Magboltz program [7] and $l_{d}$ is the drift length. Using numerical methods we solve Eq. 1 and find the best estimate of the track coordinate $x_{t}$.

The uncertainty $\sigma_{x}$ (fig. 6) of the track coordinate $x_{t}$ is estimated in the limit $\Delta_{p} / w_{t} \gg 1$ taking into account the binomial fluctuations of the number of primary electrons collected by the two pads [8]:

$$
\sigma_{x}=\sqrt{2 \pi} w_{t} e^{\frac{\left(x_{t}-x_{p}\right)^{2}}{2 w_{t}^{2}}} \sqrt{\frac{f(1-f)}{\alpha N_{p}}}
$$

where $f=\frac{q}{q+q^{\prime}}, N_{p}$ is the number of primary electrons in the cloud estimated from the total collected charge $\left(q+q^{\prime}\right)$ and $\alpha \simeq 0.5$ is a reduction factor to take into account gain fluctuations. Indeed gain fluctuations due to the stochastic nature of the avalanche process reduce the number of effective primary electrons to be considered in the uncertainty evaluation. The pull distribution (fig. 6) of the cluster position with respect to the fit position of the track has a width of approximately 1 assuming that the number of effective electrons is one half of the electrons in the cloud. This reduction factor is in fair agreement with expectations from numerical simulations [9]. A term representing the electronic noise affecting the measurement of the charges $q$ and $q^{\prime}$ is added in quadrature to the right hand side of Eq.2. 

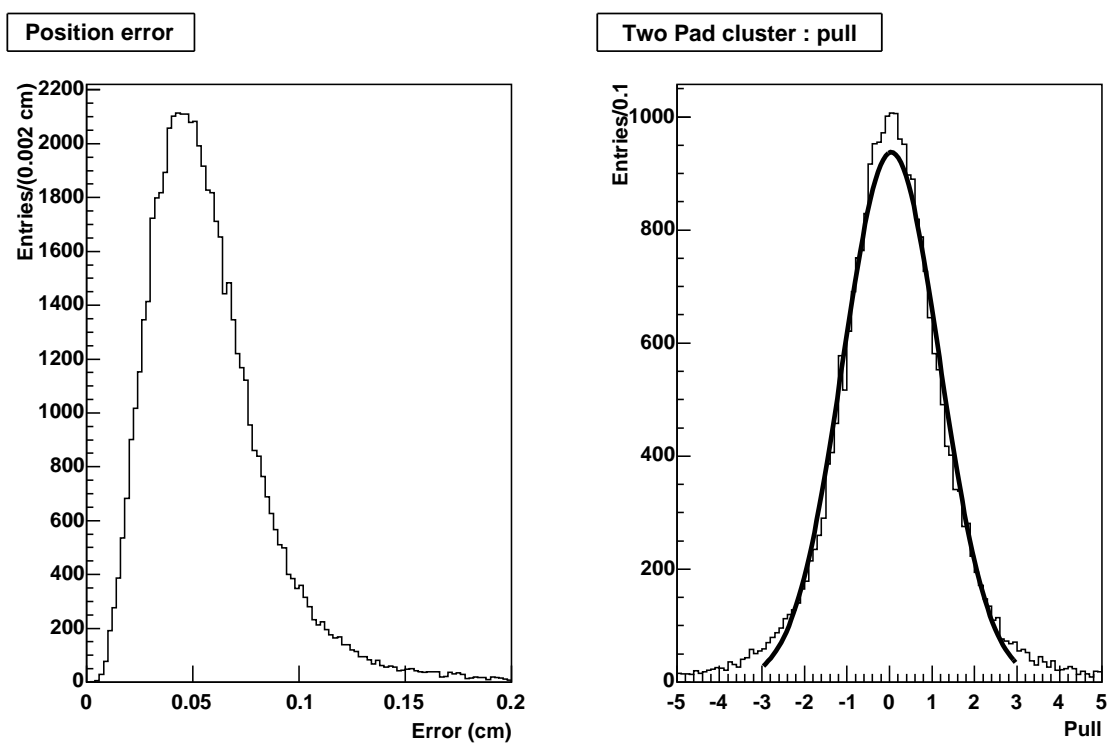

Fig. 6. Left plot: the estimated error distribution associated to clusters with two pads for $\mathrm{B}=0.2 \mathrm{~T}$. The mean value of the distribution is $570 \mu \mathrm{m}$. Right plot: the pull of the cluster position with respect to the fit position of the track. The curve represents a gaussian fit to the distribution with a width of 1.1 .

For clusters with three hits, the position is estimated in a similar way, fitting a gaussian distribution to the two measured charge ratios. We have associated an uncertainty equal to $\Delta_{p} / \sqrt{12}$ to clusters with only one hit, where $\Delta_{p}$ is the pad width.

Tracks close to the vertical axis and crossing the two Micromegas modules are reconstructed and fitted separately in the two projections (one in the readout plane, the other in the plane containing the vertical axis and the drift direction) with a least square fit. The space point resolution for each cluster has been studied considering the residual between its position and the extrapolated track position without using this clusters. Figure 7 shows the gaussian widths of these residuals for $\mathrm{B}=0.2 \mathrm{~T}$ in fair agreement with the result of the Geant 4 simulation. To estimate the momentum resolution capabilities of this device it is necessary to consider the fraction of rows with one, two or more pad hit (fig. 7): approximately $75 \%$ of the clusters have two or more hits at one meter drift length for $\mathrm{B}=0.2 \mathrm{~T}$. This is the experimental situation corresponding to the T2K TPC. The momentum resolution of a TPC equipped with these detectors and measuring a track length of $70 \mathrm{~cm}$ in a $0.2 \mathrm{~T}$ magnetic field is expected to be $8 \%$ at $1 \mathrm{GeV} / \mathrm{c}$, slightly better than the required performance of $10 \%$ for the $\mathrm{T} 2 \mathrm{~K}$ tracker.

A first study of the gas properties has been done during the data taking by studying tracks that cross the field cage cathode. These tracks give a very precise measurement of the drift velocity that is in agreement with Magboltz predictions. Clusters with two pads can be used to study the width of the electron cloud if the position given by the track fit is assumed. This is shown in figure 8 . The mean value of 

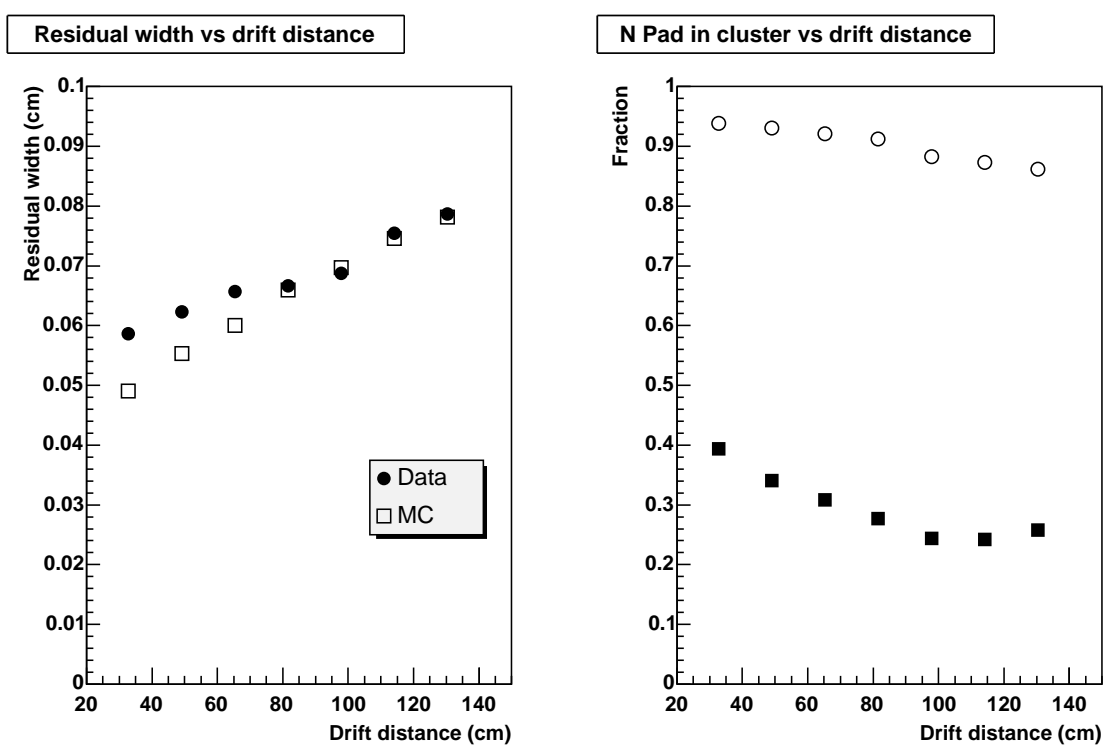

Fig. 7. Left plot: the gaussian width for the two pad cluster track residual as a function of the drift distance for $\mathrm{B}=0.2 \mathrm{~T}$. The open squares show the width estimated using a Geant 4 MC simulation. Right plot: the cumulative fraction of clusters with one pad (squares) and two pads (open circles).

this distribution as a function of the drift length $l_{d}$ has been fit with the function $\sqrt{s_{0}^{2}+C_{T} l_{d}}$. The first term models the uncertainty due to the track position. The values for the transverse diffusion coefficient $C_{T}$ are in reasonable agreement with the Magboltz predictions (table 1). The attenuation in the Ar- $\mathrm{CF}_{4}$-Isobutane mixture was found to be negligible after a few days of gas flow with a lower limit on the attenuation length of $30 \mathrm{~m}$.

Table 1

Expected and measured (last two columns) gas properties (drift velocity $v_{d}(\mathrm{~cm} / \mu \mathrm{s})$ and transverse diffusion coefficient $C_{T}(\mu \mathrm{m} / \sqrt{\mathrm{cm}})$ ) for different experimental conditions.

\begin{tabular}{|c|c|c|c|c|}
\hline Field & $v_{d}(\exp )$ & $C_{T}(\exp )$ & $v_{d}$ (meas) & $C_{T}$ (meas) \\
\hline $\mathrm{B}=0 \mathrm{~T} \mathrm{E}=160 \mathrm{~V} / \mathrm{cm}$ & 6.50 & 309 & $6.26 \pm 0.05$ & $302 \pm 15$ \\
$\mathrm{~B}=0.2 \mathrm{~T} \mathrm{E}=160 \mathrm{~V} / \mathrm{cm}$ & 6.50 & 237 & $6.27 \pm 0.05$ & $253 \pm 15$ \\
$\mathrm{~B}=0.4 \mathrm{~T} \mathrm{E}=160 \mathrm{~V} / \mathrm{cm}$ & 6.50 & 157 & $6.30 \pm 0.05$ & $173 \pm 15$ \\
$\mathrm{~B}=0.4 \mathrm{~T} \mathrm{E}=100 \mathrm{~V} / \mathrm{cm}$ & 4.46 & 157 & $4.23 \pm 0.02$ & $176 \pm 15$ \\
\hline
\end{tabular}

A first study of the measurement of $\mathrm{dE} / \mathrm{dx}$, the energy loss per unit length, has been performed considering for each track the total charge detected in each pad row. A truncated mean retaining only $80 \%$ samples with lower measured charge has been used. This mean has been corrected for the track length in the active volume of the TPC. The results are shown in figure 9. A resolution on the $\mathrm{dE} / \mathrm{dx}$ of $12.2 \pm 0.4 \%$ (Fig. 9) has been obtained for a nominal track length of $38.4 \mathrm{~cm}$ and 48 samples. Using the PDG parametrization [10], a resolution around $10 \%$ is expected for this track length. 


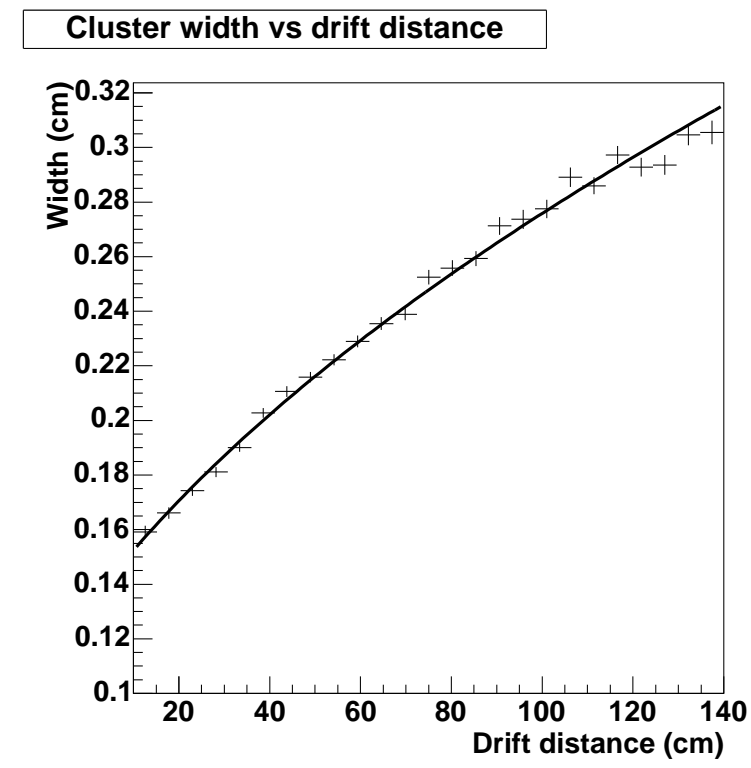

Fig. 8. Gaussian width of the electron cloud measured using the track position and the charge ratio in clusters with two pads as a function of the drift distance for $\mathrm{B}=0.2 \mathrm{~T}$. The curve shows the result of the fit to the function $\sqrt{s_{0}^{2}+C_{T} l_{\text {drift }}}$ where the transverse diffusion coefficient $C_{T}$ has been found $253 \mu \mathrm{m} / \sqrt{\mathrm{cm}}$.
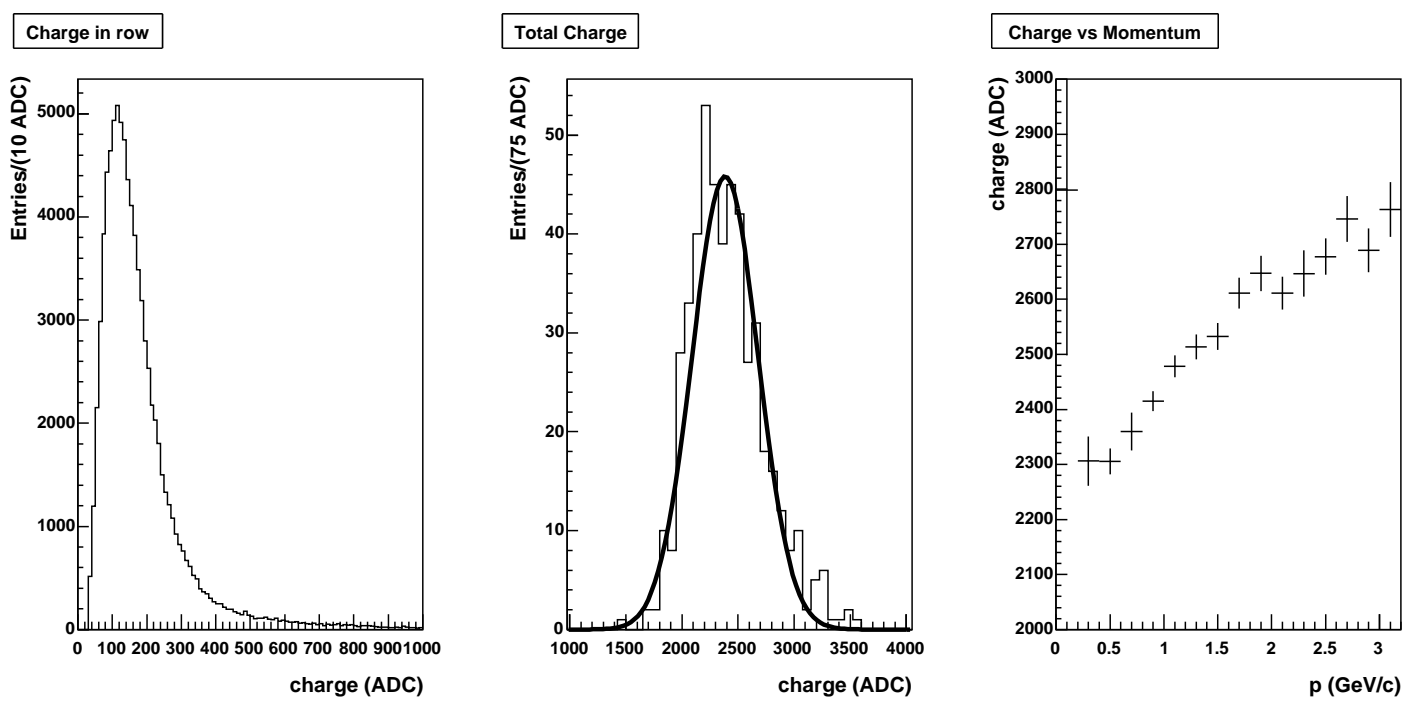

Fig. 9. Left plot: the charge per pad row. Middle plot: the $80 \%$ truncated mean for the sum of the charge in the two detectors. A resolution of $12.2 \%$ has been obtained. Right Plot: the measured charge versus the track momentum.

\section{Conclusion}

We have built four large bulk Micromegas detectors as prototypes for the T2K TPC. We have tested these detectors in the former HARP field cage setup with a magnetic field. Cosmic ray data have been acquired in a variety of experimental 
conditions. The detector has shown good performances in terms of gain and space point resolution.

\section{Acknowledgements}

We wish to warmly thank Luciano Musa for his kind assistance in setting up and operating the electronic chain and the DAQ software.

\section{References}

[1] I. Giomataris et al., Nucl. Instr. and Meth. A560 (2006) 495.

[2] Gantois, B.P. 307, F-88105 Saint Die des Vosges cedex, France.

[3] DuPont Electronic Materials, 14 Alexander Drive - Research Triangle Park, NC $27709-4425$.

[4] M.G. Catanesi et al., "The HARP detector at the CERN PS", to be published in Nucl. Instr. and Meth. A.

[5] L. Musa et al., Proc. of the IEEE Nucl. Sc. Symp., Portland (2003); http://ep-ed-alicetpc.web.cern.ch

[6] S. Agostinelli et al., Nucl. Instr. and Meth. A506 (2003) 250.

[7] S. Biagi, Nucl. Instr. and Meth. A421 (1999) 234.

[8] D. Karlen et al., Nucl. Instr. and Meth. A555 (2005) 80 (Appendix A).

[9] M. Kobayashi, Nucl. Instr. and Meth. A562 (2006) 136.

[10] W.M. Yao et al., J. Phys. G 33 (2006) 283. 